\title{
Planetary nebulae and star formation history in the Galactic disk and bulge
}

\author{
Yulia Milanova ${ }^{1}$ and Alexander Kholtygin ${ }^{1}$ \\ ${ }^{1}$ Astronomical Institute of St.Petersburg State University, Russia email: \\ yulia.milanova@gmail.com
}

\begin{abstract}
The modern observations of the planetary nebulae (PNe) were used to create a new catalogue of $\mathrm{He}, \mathrm{C}, \mathrm{N}, \mathrm{O}, \mathrm{Ne}, \mathrm{S}$ and some other element abundances for more than 300 Galactic and extragalactic PNe. This catalogue was a start point for studying the star formation history in the Galactic disk. We have found that $\mathrm{He}, \mathrm{C}, \mathrm{N}$ and $\mathrm{O}$ abundances in the thin disk PNe are very close to those in bulge PNe. We also established that the abundances of the same elements in the PNe belonging the halo of the Milky Way and the Magellanic Clouds are similar. We have estimated the mean ages of the progenitors of PNe in the thin and thick disks and in the bulge. The evidences in the favor of the suggestion that the formation of the intermediate-mass star began in bulge and only then continued in the thin disk were found.
\end{abstract}

Keywords. galaxies: evolution, planetary nebulae: general, Magellanic Clouds

\section{Introduction}

The PN phenomenon is an intermediate phase between the stars and interstellar medium. So the research of the properties and chemical abundances in the ensemble of Galactic and Magellanic Cloud's planetary nebulae open us a possibility to study of star formation history and the chemical evilution of the Milky Way. The following problems are considered:

- The comparison of the chemical abundance of the PNe ensemble with the synthetic models of the LIMS evolution.

- An evolution status of the PNe with the extremely low element abundances.

- A comparison of the element abundances in the different galactic subsystems.

- A comparison of the abundances for nebulae in our Galaxy and in the Magellanic Clouds.

- Determination of abundance gradients for the Milky Way PNe ensembles both for all galactic PNe and for nebulae with the different ages of their progenitors.

- Investigation of the evolution of the element abundances in the different regions of the Milky Way in a comparison with the models of the Galaxy chemical evolution.

\section{Model and catalog of atomic data}

The modern observations of the planetary nebulae $(\mathrm{PNe})$ were used by us to recalculate the abundances and other parameters of more than $120 \mathrm{PNe}$ of the Milky Way and Magellanic Clouds with taking into account the temperature fluctuations inside the nebulae (Milanova \& Kholtygin, 2009). We use the stochastic model of the nebulae descibed by Kholtygin (1998) to take into account the temperature and density fluctuations and the real distributions for the errors in the observed intensities. The atomic data were taken from the catalogue Golovatyj et al. (1997) and from the literature. We compare in 

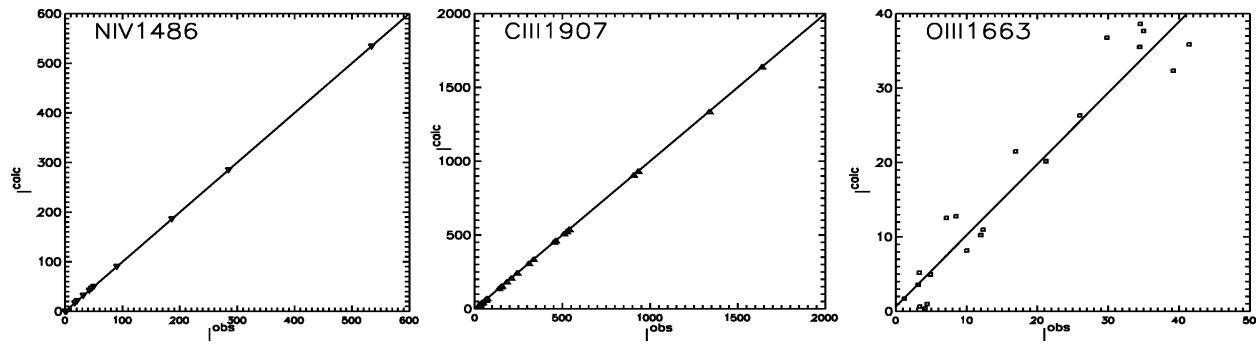

Figure 1. A comparison of observed $\left(I^{o b s}\right)$ and calculated intensities of UV lines in spectra of selected PNe (in a scale $I\left(\mathrm{H}_{\beta}\right)=100$ ). Left panel: NIV $\lambda$ 1486, center panel: CIII $\lambda 1907$ and right panel: NIV $\lambda 1486$.

Fig. 1 the calculated and observed UV line intensities in spectra of planetary nebulae to demonstrate a good quality of our model fit.

\section{Magellanic Clouds PNe}

At present, a large number of extragalactic PNe are accessible to spectroscopic observations. We model the high quality spectra of for the nearest (LMC and SMC) extragalactic nebulae by using the above decribed procedure.
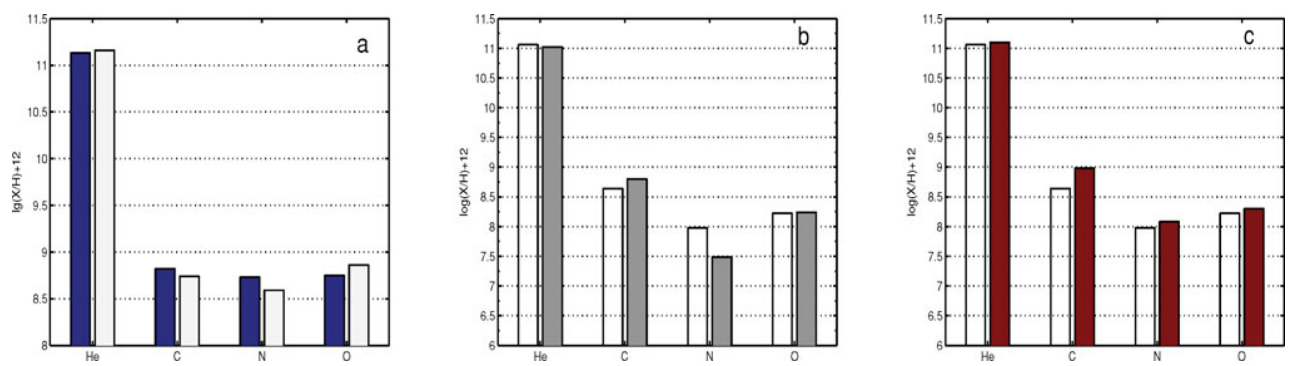

Figure 2. Comparison of the $\mathrm{He}, \mathrm{C}, \mathrm{N}$, and $\mathrm{O}$ abundances in $\mathrm{PNe}$ of various Galactic subsystems: (a) Galactic type IIa PNe (dark columns) and bulge nebulae (light columns); (b) the same as

(a) for PNe of the Galactic halo and the LMC; (c) the same as (b) for SMC nebulae.

In figure $2(\mathrm{~b}, \mathrm{c})$ we compare the $\mathrm{He}, \mathrm{C}, \mathrm{N}$, and $\mathrm{O}$ abundances in $\mathrm{PNe}$ of the Galactic halo and the Magellanic Clouds. A similarity between the elemental abundances in these objects can be seen. It can be the indicator of their close evolutionary status.

\section{A new catalogue of the parameters of the PNe}

The calculated by us parameters of PNe together with data taken from the literature are used to create a new catalogue of galactic and extragalactic PNe parameters for more than 300 objects of the Milky Way and Magellanic Clouds. The electron version of the catalogue is located at the URL http://www.astro.spbu.ru/staff/afk/GalChemEvol.html.

\section{Galactic bulge formation}

Many details of the bulge formation and its connection with other galactic subsystems in particular the thin and thick disks remain unclear. To know which PNe of the Galactic 
disk are similar to bulge objects we compare the He, C, N, and $\mathrm{O}$ abundances in bulge and disk nebulae.
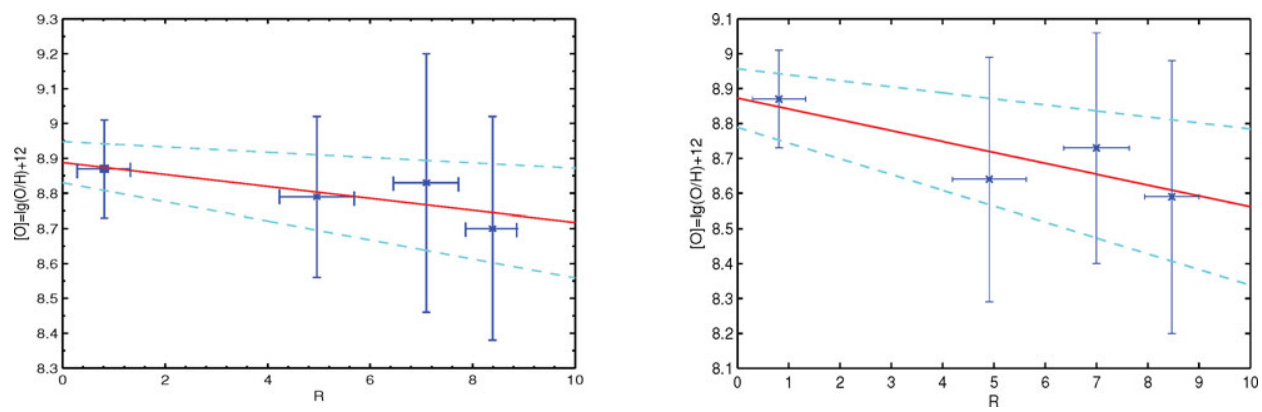

Figure 3. (a) Averaged oxygen abundances in type IIa (asterisks) and bulge (filled square) nebulae vz. their mean Galactocentric distance. The solid line gives a linear fit to this dependence, the dotted lines shows one standard deviation from the fit. (b) The same as Fig. (a), but for all type II nebulae.

The He, $\mathrm{C}, \mathrm{N}$ and $\mathrm{O}$ abundances in the thin disk PNe (type IIa in the modified Peimbert's classification given by Quireza et al., 2007) appeared to be close to those for bulge PNe (see Fig. 2 (a)).

In this case the dependence of elemental abundances in $\mathrm{PNe}$ on the galactocentric distance $\mathrm{R}$ should must be continuous from thin disk to bulge. To test this assumption, we analyzed the radial dependence of the $\mathrm{O}$ abundance, which is almost constant during the evolution of intermediate-mass stars. As can be seen in Fig. 3 the mean oxygen abundances for bulge $\mathrm{PNe}$ continue a dependence of ratio $[\mathrm{O} / \mathrm{H}]$ for thin disk $\mathrm{PNe}$.

The oxygen abundance gradient $d[\mathrm{O} / \mathrm{H}] / d R=-0.017 \pm 0.01 \mathrm{dex} \mathrm{kpc}^{-1}$, when only the type IIa nebulae are included in the sample. It is slightly higher than a value -0.012 dex $\mathrm{kpc}^{-1}$ obtained by Kholtygin and Milanova (2006) from the analysis of the O abundance for all Galactic disk PNe. The value of $d[\mathrm{O} / \mathrm{H}] / d R=-0.031 \pm 0.014 \mathrm{dex} \mathrm{kpc}^{-1}$ For all type II PNe. The type IIa nebulae are thin disk objects and they have an intermediate age of their progenitor stars, which is equal to 4-6 Gyr from the present epoch (Quireza et al. 2007). We propose that the bulge is formed on the early stage of the Galaxy evolution. After that the stellar formation begins in the disk starting from the regions close to bulge.

We compare the mean radial abundance gradients for disks of spiral galaxies in the table 1. We can see from the table that our values for the Milky Way disk are in a good agreement with those for galaxy M33, while our abundance gradient for thin Galactic disk $\mathrm{PNe}$ is similar to those for the Andromeda galaxy.

\section{Extremely low abundance PNe}

There exist an important group of stars in our Galaxy with low initial element abundances borned at the early stages of the Galaxy evolution. To select the progenitors of the PNe with low initial element abundances we consider the $\mathrm{O}, \mathrm{Ne}, \mathrm{S}, \mathrm{Cl}$ and $\mathrm{Ar}$ which abundances weakly changed during the progenitor star evolution. We adopt the next criteria to select the extremely low abundant progenitors of PNe: the mettallicity $[X]_{\odot}<-1.0$ for one ore more elements or $[X]_{\odot}<-0.5$ for at least two elements .

The distribution of ratios $[\mathrm{He} / \mathrm{H}]$ for these nebulae is presented in Fig. 4 (left). We can conclude that for PNe with low element abundances this ratio is about of 1.5 times of the solar value of this ratio. In Fig. 4 (right) are shown the positions on the HR diagram 
Table 1. Radial abundance gradients for disks of the spirals.

\begin{tabular}{lccl}
\hline Galaxy Name & $\mathrm{d}[\mathrm{O} / \mathrm{H}] / \mathrm{dR}(\mathrm{dex} / \mathrm{kpc})$ & $\mathrm{d}[\mathrm{Ne} / \mathrm{H}] / \mathrm{dR}(\mathrm{dex} / \mathrm{kpc})$ & Reference \\
\hline M31 & -0.03 & - & Garnett (1997) \\
M33 & $-0.012 \pm 0.011$ & $-0.016 \pm 0.017$ & Crockett et al., 2006 \\
M51 & -0.046 & - & Garnett (1997) \\
M81 & -0.08 & - & Garnett (1997) \\
M101 & $-0.028 \pm 0.01$ & - & Cedrés et al., 2004 \\
NGC 2403 & $-0.102 \pm 0.009$ & - & Garnett (1997) \\
Milky Way & & & Lunyova \& \\
(disk) & -0.012 & -0.019 & Kholtygin (2002) \\
Milky Way & & - & \\
(thin disk) & -0.031 & - & Present work \\
\hline
\end{tabular}
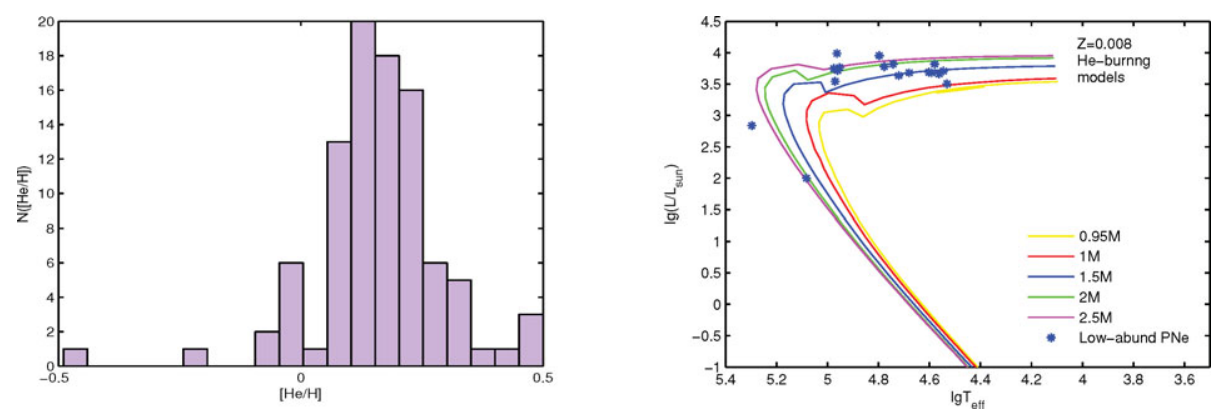

Figure 4. Left panel: distribution of $[\mathrm{He} / \mathrm{H}]$ abundance ratio for $\mathrm{PNe}$ with low abundances. Right panel: low abundances PNe central stars (asterisks) on a H-R diagram. Tracks are taken from He-burning star evolutionary model by Vassilidias \& Wood (1994) with initial metallicity $Z=0.008$. Initial masses of stars (from bottom to top) are equal to 0.95, 1.0, 1.5, 2.0 and 2.5 solar masses.

the low abundant PNe. We see the concentration of the central stars of the considered $\mathrm{PNe}$ in the region of the low masses of the progenitors.

This study was supported by a grant from the President of Russia (NSh-1318.2008.2) for "Support of Leading Scientific Schools".

\section{References}

Cedrés, B., Urbaneja, M. A., \& Cepa, J. 2004, A $\& A, 422,511$

Crockett, N. R., Garnett, D. R., Massey, P., \& Jacoby, G. 2006, Ap. J., 637(2), 741

Garnett, D. R., Shields, G. A., Skillman, G. A., et al. 1997, Ap. J., 489, 63

Golovatyj V. V., Sapar A., Feklistova T., \& Kholtygin A. F. 1997, Catalogue of atomic data for low-density astrophysical plasma, Astron. Astroph. Trans., 12, 85

Kholtygin, A. F. 1998, A\&A, 329, 691

Lunyova, Yu. V. \& Kholtygin, A. F. 2002, Astrophysics (English translation of Astrofizika), $45(3), 370$

Milanova, Yu. V. \& Kholtygin, A. F. 2006, Astronomy Letters, 32, 557

Milanova, Yu. V. \& Kholtygin, A. F. 2009, Astronomy Letters, 35, 563

Quireza, C., Rocha-Pinto H. J., \& Maciel, W. J. 2007, A\&SA, 475, 217

Vassilidias, V. \& Wood, P. R. 1994, Ap. J. Suppl. Ser., 92, 125 